\title{
BMJ Open Double blind, randomised, placebo- controlled trial to evaluate the efficacy of esomeprazole to treat early onset pre-eclampsia (PIE Trial): a study protocol
}

\author{
Catherine A Cluver, ${ }^{1,2}$ Susan P Walker, ${ }^{3}$ Ben W Mol, ${ }^{4}$ Gerard B Theron, ${ }^{1}$ \\ David R Hall, ${ }^{1}$ Richard Hiscock, ${ }^{5}$ N Hannan, ${ }^{2,3}$ S Tong $^{2,3}$
}

To cite: Cluver CA, Walker SP, Mol BW, et al. Double blind, randomised, placebo-controlled trial to evaluate the efficacy of esomeprazole to treat early onset pre-eclampsia (PIE Trial): a study protocol. $B M J$ Open 2015;5:e008211. doi:10.1136/bmjopen-2015008211

- Prepublication history and additional material is available. To view please visit the journal (http://dx.doi.org/ 10.1136/bmjopen-2015008211).

Received 16 March 2015 Revised 1 September 2015 Accepted 6 October 2015

CrossMark

For numbered affiliations see end of article.

Correspondence to Dr Catherine A Cluver; cathycluver@hotmail.com

\section{ABSTRACT}

Introduction: Pre-eclampsia is a major complication of pregnancy, globally responsible for 60000 maternal deaths per year, and far greater numbers of fetal losses. There is no definitive treatment other than delivery. A drug that can quench the disease process could be useful to treat early onset pre-eclampsia, as it could allow pregnancies to safely continue to a gestation where fetal outcomes are significantly improved. We have generated preclinical data to show esomeprazole, a proton pump inhibitor used for gastric reflux, has potent biological effects that makes it a worthwhile therapeutic candidate. Esomeprazole potently decreases soluble fms-like tyrosine kinase 1 (sFlt-1) and soluble endoglin secretion from placenta and endothelial cells, and has biological actions to mitigate endothelial dysfunction and oxidative stress.

Methods and analysis: We propose undertaking a phase II, double blind, randomised controlled clinical trial to examine whether administering $40 \mathrm{mg}$ esomeprazole daily may prolong gestation in women with early onset pre-eclampsia. We will recruit 120 women (gestational age of $26+0$ to $31+6$ weeks) who will be randomised to receive either esomeprazole or an identical placebo. The primary outcome will be the number of days from randomisation to delivery. Secondary outcomes include maternal, fetal and neonatal composite and individual outcomes. Maternal outcomes include maternal death, eclampsia, pulmonary oedema, severe renal impairment, cerebral vascular events and liver haematoma or rupture. Neonatal outcomes include neonatal death within 6 weeks after the due date, intraventricular haemorrhage, necrotising enterocolitis and bronchopulmonary dysplasia. We will examine whether esomeprazole can decrease serum sFlt- 1 and soluble endoglin levels and we will record the safety of esomeprazole in these pregnancies.

Ethics and dissemination: This study has ethical approval (Protocol V.2.4, M14/09/038, Federal Wide assurance Number 00001372, IRB0005239), and is registered with NHREC (ID 3649) and the Pan African Clinical Trial Registry (PACTR201504000771349). Data will be presented at international conferences and published in peer-reviewed journals.

\section{Strengths and limitation of this study}

- This is a protocol for a randomised, double blind, placebo controlled clinical trial.

- This is the first trial to assess whether esomeprazole is a treatment option for pre-eclampsia.

- We plan to recruit 120 participants and we have designed this study to be sufficiently powered to identify a prolongation of pregnancy.

- It may be underpowered to show improvements in maternal and perinatal outcomes. Therefore, if the trial yields a positive result, a larger subsequent multicentre study may be needed.

\section{INTRODUCTION}

Pre-eclampsia is one of the most serious complications of pregnancy, affecting $3-8 \%$ of pregnancies worldwide and is a leading cause of maternal and fetal/neonatal morbidity. ${ }^{1-3}$ Pre-eclampsia is estimated to cause more than 60000 maternal deaths annually. ${ }^{4}$ There is no treatment that can quench the disease progression and the only treatment option available to arrest the disease is delivery of the pregnancy. ${ }^{5}$ For pre-eclampsia occurring at preterm gestations, clinicians are often forced to deliver early on maternal indications to prevent major maternal morbidity, but in doing so, inflict severe prematurity on the fetus. In particular, fetuses delivered at less than 33 weeks' gestation are at significant risk of severe disability including cerebral palsy, stroke (intracerebral bleeding), retinopathy of prematurity, chronic lung disease and death. ${ }^{6}$

If an affordable and safe treatment was available that could temporise the disease progression of pre-eclampsia, clinicians could safely delay delivery and gain gestation to improve fetal outcome. This could save the lives of many infants and decrease the hospital burden caused by iatrogenic 
prematurity. Such a treatment would be in keeping with the United Nations Millennium Development Goals to reduce child mortality and improve maternal health. ${ }^{8}$

The pre-eclamptic placenta releases antiangiogenic soluble fms-like tyrosine kinase 1 (sFlt-1) and soluble endoglin (sEng) into the maternal circulation. These factors are responsible for causing widespread maternal endothelial dysfunction and organ injury seen in clinical disease. ${ }^{9}$ In addition, pre-eclampsia is strongly associated with placental and systemic oxidative stress.

Esomeprazole is a proton pump inhibitor widely used to treat women with gastric reflux in pregnancy. Large observational studies including administration during the first, second and third trimesters have not identified associations with adverse pregnancy outcomes, notably teratogenesis. $^{10-12}$

We have performed preclinical laboratory studies where we have identified esomeprazole as a promising candidate therapeutic for pre-eclampsia. Esomeprazole potently decreased sFlt-1 and sEng secretion from placenta and endothelial cells, has strong actions mitigating endothelial dysfunction and has antioxidant properties. (A manuscript reporting this preclinical data has been submitted elsewhere and this work was recently presented.) ${ }^{13}$

\section{OBJECTIVES}

The primary objective is to examine whether a single daily dose of $40 \mathrm{mg}$ of esomeprazole can safely prolong gestation in women with early onset pre-eclampsia diagnosed $26+0-31+6$ weeks who are being managed expectantly, compared with expectant management alone.

The secondary objectives are to determine whether esomeprazole can improve maternal, fetal and neonatal outcomes, and to determine whether esomeprazole can significantly decrease levels of circulating sFlt-1 and/or

\section{Box 1 Inclusion criteria}

A diagnosis of one of the following:

- Pre-eclampsia ${ }^{14}$

- Gestational hypertension with evidence of pre-eclampsia

- Pre-existing hypertension with evidence of pre-eclampsia

- Unclassified proteinuric hypertension

AND

All of the following is present:

- Gestational age between $26+0$ and 31+6 weeks

- Estimated fetal weight by ultrasound between 500 and $1800 \mathrm{~g}$ (if gestation is not certain)

- Singleton pregnancy

- The managing clinicians have made the assessment to proceed with expectant management and that delivery is not expected within $48 \mathrm{~h}$

- The managing clinician and neonatologist believe that the fetus could potentially be delivered in a viable condition

- No suspicions of a major fetal anomaly or malformation

- Patient will be admitted to hospital for expectant management and standardised care
sEng. Furthermore, we will examine whether esomeprazole is safe and well tolerated in the mother and infant.

\section{METHODS}

The full protocol is included as supplementary information (see online supplementary information 1).

\section{Study design}

Phase II hospital-based, double blind, randomised, placebo-controlled trial.

\section{Study population}

Pregnant women diagnosed with early onset pre-eclampsia at a gestational age between $26+0$ and $31+6$ weeks at Tygerberg hospital (Western Cape Provence of South Africa) will be invited to participate. To be enrolled, the treating team needs to have determined after their initial assessment that delivery is unlikely to be required within $48 \mathrm{~h}$. A starting point of $26+0$ weeks has been chosen as this would be the earliest gestation that Tygerberg Hospital would consider to be viable and are suitable to be offered expectant management.

\section{Inclusion criteria}

We will recruit women with a singleton pregnancy diagnosed with pre-eclampsia, defined according to the criteria published by the International Society for the Study of Hypertension in Pregnancy (ISSHP). ${ }^{14}$

We will seek to recruit those with pregnancies at a gestational age between $26+0$ and $31+6$ weeks, determined by either period dates (if the women is certain of her last menstrual period) or by an early, or mid trimester pregnancy ultrasound. If the gestational age is uncertain, we will recruit participants with an estimated fetal weight between 500 and $1800 \mathrm{~g}$, determined by ultrasound performed at presentation. At Tygerberg Hospital we would not consider a pregnancy to be viable under $500 \mathrm{~g}$ and we would not offer expectant management if the fetal weight was above $1800 \mathrm{~g}$.

To be eligible for this study, the treating clinicians need to have made an initial assessment and deemed that the patient is suitable for expectant management, that the fetus would benefit from expectant management and that immediate delivery is not required. A full list of the inclusion criteria is shown in box 1 .

\section{Exclusion criteria}

Exclusion criteria include women with established maternal or fetal compromise that necessitates delivery, the current use of a proton pump inhibitor, contraindications to the use of a proton pump inhibitor or the use of medications that may interact with proton pump inhibitors. A full list of the exclusion criteria is shown in box 2 .

\section{Randomisation and allocation concealment}

Randomisation will be done in an equal ratio of esomeprazole to placebo. An online, web-based sequence 
Box 2 Exclusion criteria

Any of the following at the initial assessment:

- Patient is unable or unwilling to give consent

- Established fetal compromise that necessitates delivery

- The presence of: Eclampsia

- Severe hypertension

- Cerebrovascular event

- Posterior reversible encephalopathy syndrome

- Severe renal impairment

- Pulmonary oedema

- Left-sided heart failure

- Disseminated intravascular coagulation

- Platelet count $<50 \times 10^{9}$

- Haemolysis, elevated liver enzymes and low platelets syndrome

- Liver transaminases $>500 \mathrm{IU} / \mathrm{L}$

- Liver haematoma or rupture

- Severe ascites

Current use of a proton pump inhibitor

- Contraindications or a hypersensitivity reaction to the use of a proton pump inhibitor

- Current use of a drug that may be affected by a proton pump inhibitor

generator system will be used. It will be linked with codes for placebo and treatment tablets provided by the manufacturer contracted to produce the trial medication. Both the researchers and participants will be blinded.

The gestational age at diagnosis is likely to affect allowable length of pregnancy prolongation. To ensure treatment group allocation is balanced for this potential variable, we will stratify randomisation into two strata based on gestational age. Strata 1 includes a gestational age of 26+0 up to and including 28+6 weeks (500-1200 g if gestation is unknown). Strata 2 includes a gestational age of $29+0$ up to and including $31+6$ weeks (1200$1800 \mathrm{~g}$ if gestation is unknown). Thus, randomisation will include blocking within each gestational age stratum. We propose using blocks of 4-6 with the size and order randomly assigned.

Once the participants have been randomised, the treatment pack with the same code will be allocated to the participant. All treatment packs will be identical and will contain either active tablets or placebo. The researchers will have no access to the randomisation list. This process will ensure that there is allocation concealment throughout the conduct of the trial.

\section{Participant enrolment}

Participants will be identified after they have been admitted to Tygerberg Hospital (tertiary referral centre) with a diagnosis of early onset pre-eclampsia for expectant management. An information leaflet will be given to all potential participants and informed consent will be obtained (see online supplementary information 2 and 3). Each participant will be given an individual treatment pack containing either esomeprazole or placebo which will be produced by a contracted manufacturer IDT pharmaceuticals (http://en.idtaus.com.au). Labelling, storage and preparation will be done according to the requirements of the Medicines for Human Use (Clinical Trials) regulations.

\section{Intervention}

Participants will be randomised to daily administration of either active tablets containing $40 \mathrm{mg}$ of esomeprazole or an identical placebo tablet orally once a day. Participants will remain under the care of the hospital treating team, and the study will not alter or interfere with the care given routinely to women with early onset pre-eclampsia, including on when to deliver.

\section{Routine expectant management for pre-eclampsia}

Expectant management for early onset pre-eclampsia involves admission to hospital, and close maternal and fetal surveillance. Maternal surveillance includes four hourly blood pressure measurement, twice daily clinical assessment, daily urinalysis and twice weekly assessments with blood tests (full blood count, renal function tests and hepatocellular enzymes if haemolysis, elevated liver enzymes and low platelets syndrome is suspected) and $24 \mathrm{~h}$ urinary protein measurement on admission. Fetal surveillance includes ultrasound assessments to assess growth of the fetus, the amniotic fluid index and fetal well-being including Doppler velocimetry of the umbilical artery, the ductus venosus and the middle cerebral artery. If there are no signs of fetal growth restriction or fetal compromise, the ultrasound is repeated two weekly to ensure there has been adequate trajectory of fetal growth. If there are signs of fetal growth restriction or fetal compromise, the frequency of ultrasound surveillance will be increased. Six hourly cardiotocographs are performed to assess the ongoing fetal condition. We will follow the Tygerberg Hospital protocols to monitor preterm fetal growth restriction and delivery may occur on fetal grounds if required.

All participants will receive two doses of $\beta$-methasone $24 \mathrm{~h}$ apart to reduce the risks of neonatal respiratory distress syndrome, intracranial haemorrhage and necrotising enterocolitis. A single repeat dose is usually given 1 week later. Most participants will be on antihypertensive treatment with the aim to stabilise the systolic blood pressure between 140 and $150 \mathrm{~mm} \mathrm{Hg}$ and the diastolic blood pressure between 90 and $100 \mathrm{~mm} \mathrm{Hg}$. The medications used to treat the blood pressure will be documented. All women should already be receiving calcium, iron and folic acid supplementation.

Clinical care will be left up to the discretion of the clinical team. The indication for delivery will be a clinical decision. Indications for delivery may include failure to control blood pressure, the development of major maternal or fetal complications, or intrauterine fetal death. Expectant management will usually end at a gestation of 34 weeks. 
Sample size and stratification according to gestation at recruitment

The reported duration that fetuses remain in utero after diagnosis of preterm pre-eclampsia is a mean of 11 days (SD of 7 days) and a median 9 days (range of 1-47 days). ${ }^{15}$ These data are derived from a descriptive study on expectant management of early onset pre-eclampsia at Tygerberg Hospital, where we propose to undertake this study ${ }^{15}$ For $90 \%$ power, with a two-sided $\alpha$ set of $0.05,43$ patients are required in each group to identify a gain in gestation of 5 days. Given these data were skewed, the sample size has been multiplied by 1.15 to statistically correct for non-normality. ${ }^{16}$ This increases the number required to 50 per arm. An additional 10 per arm will be added to allow for drop-outs. Thus, a total of 120 participants will be recruited to provide sufficient power to examine our primary outcome. (Power calculation performed using PASS V.12 software. Hintze, J. (2013). PASS 12. NCSS, LLC. Kaysville, Utah, USA).

\section{Sample collection}

Blood samples will be routinely collected twice a week. Two sets of specimens will be drawn, the routine preeclamptic monitoring samples and the trial samples. The routine blood samples include measurements of the haemoglobin, the platelet count and the urea and creatinine levels. These will be used by the managing clinicians to determine disease severity and may trigger delivery. The second set of samples will be the trial blood samples. These samples will be stored and will only be measured after delivery of the patient. These include measurements of sFlt, sEng and endothelin 1. These results will not be made available to the managing clinicians and will not affect management.

There are few data available on the pharmacokinetics of esomeprazole in pregnancy. In healthy males, the plasma elimination half-life is approximately $1-1.5 \mathrm{~h}$ and the peak plasma concentration occurs within $1-4 \mathrm{~h}$ after dosing. We propose to perform pharmacokinetic testing on a subgroup to determine if there are differences in the pregnant population. Fifteen patients in each group will undergo pharmacokinetic testing, so that blinding and allocation concealment is not affected. Blood will be drawn from an indwelling catheter in a forearm vein at $5 \mathrm{~min}$ before the medication is given (reference sample) and then at the following dosing interval: 15, 30 and $45 \mathrm{~min}$ and then at 1, 1, 5, 2, 4, 8 and $24 \mathrm{~h}$ after the initial dose is given. The sampling will be repeated on day 5 .

Urine samples will be collected two times per week and sent for spot protein: creatinine ratios. $24 \mathrm{~h}$ protein excretion is routinely measured only once on admission but for the purposes of this study we will repeat it weekly. Cord blood and placental samples will be collected at delivery.

\section{Withdrawal from the study}

All participants will be informed that they are free to withdraw from the study at any time, and that this will not affect their clinical care. Basic clinical data and samples already collected will be included in the analyses in accord with the consent obtained at trial entry.

\section{Duration of the trial}

It is anticipated that the study can be completed in approximately 4 years (2015-2018). This study has been registered with NHREC (South African Human Research Ethics Committee) and PACTR (Pan African Clinical Trials Registry).

\section{Ethical approval and dissemination}

Data will be presented at international conferences and published in peer-reviewed journals.

\section{Confidentiality}

Patient confidentiality will be protected according to the regulations set forth by Stellenbosch University's Human Research Ethics Committee or Institutional Review Board (IRB).

\section{OUTCOMES}

The primary outcome is to examine whether $40 \mathrm{mg}$ of daily esomeprazole can prolong a pregnancy complicated by early onset pre-eclampsia. We have powered the study for a further 5-day prolongation. This surrogate primary outcome marker has been chosen as it provides a sample size that is feasible and attainable for a phase II trial to test efficacy. Powering the trial to neonatal outcomes would require far greater numbers. If esomeprazole is proven to be effective, this trial would then form the basis for a larger multicentre trial powered to detect meaningful improvements in neonatal outcomes.

The secondary outcomes include maternal, fetal and neonatal mortality and morbidity, maternal serum biomarkers (including sFlt, sEng and endothelin 1) and placental samples (see boxes 3-5 for more information regarding secondary outcomes). We will present a composite outcome of the important maternal outcomes which include maternal death, eclampsia, pulmonary oedema, severe renal impairment or the need for dialysis, cerebral vascular event and the development of a liver haematoma or rupture. Other maternal outcomes will be compared as individual outcomes as listed in box 3 . We will present a composite outcome for fetal outcomes. This will include poor prognostic signs on ultrasound, significant changes on the cardiotocograph that necessitate delivery, intrauterine fetal demise and growth restriction (see box 4). The composite outcome for neonatal outcomes will include neonatal death within 6 weeks after the due date, severe intraventricular haemorrhage, necrotising enterocolitis or bronchopulmonary dysplasia. Individual neonatal outcomes will be compared as listed in box 4 . We will measure weekly the biomarkers sFlt, sEng and endothelin 1 which are antiangiogenic factors likely to play a role in the maternal endothelial dysfunction that is central to the 


\section{Box 3 Maternal secondary outcomes}

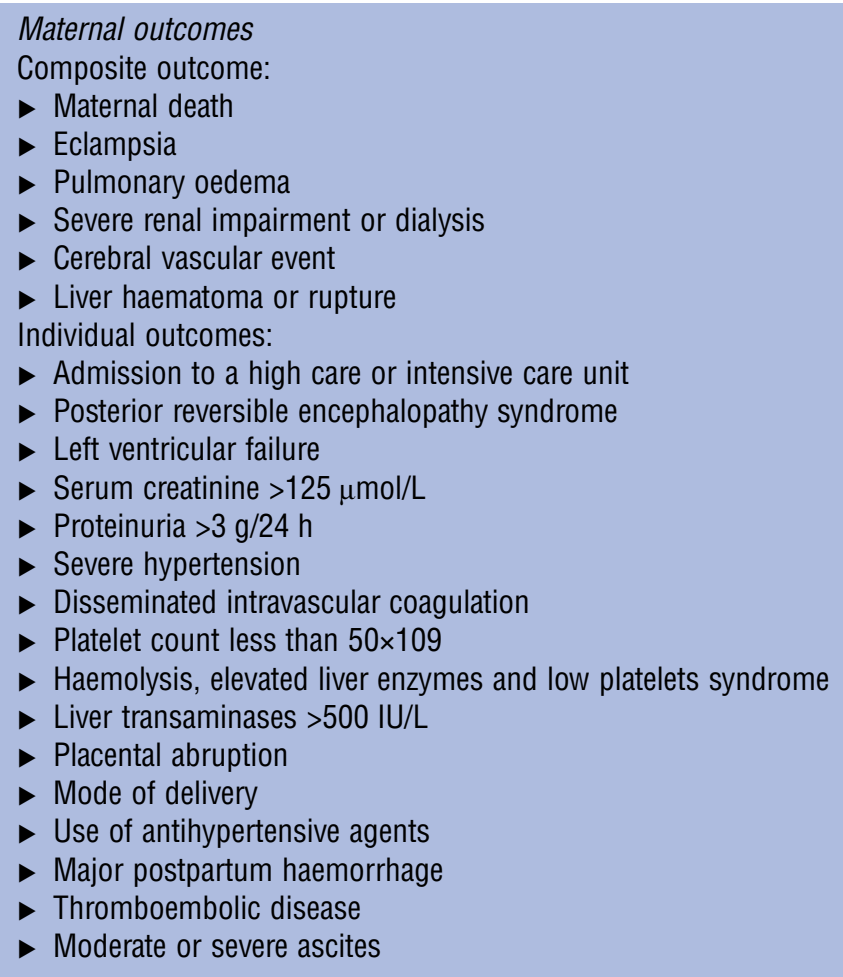

pathophysiology of pre-eclampsia (ie, it would be advantageous if treatments can decrease circulating levels of these circulating factors). Esomeprazole levels will be

\section{Box 4 Fetal and neonatal secondary outcomes}

\section{Fetal and neonatal outcomes}

Fetal composite outcome:

- Reversed $\alpha$-wave in the ductus venosus

- Significant changes in heart rate patterns

- Intrauterine fetal demise

- Fetal growth restriction

- Persistent reversed flow in the umbilical artery

- Redistribution in the middle cerebral artery

Neonatal composite outcome:

- Neonatal death within 6 weeks after the due date

- Grade III or IV intraventricular haemorrhage

- Necrotising enterocolitis

- Bronchopulmonary dysplasia

Neonatal individual outcomes:

- APGAR score $<7$ at 5 min

- Umbilical artery pH $<7.05$

- Umbilical artery lactate

- Surfactant use

- Neonatal intensive care or special care unit admission

- Intubation and mechanical ventilation

- Continuous positive airway pressure support

- Grade III/IV hyaline membrane disease

- Length of oxygen treatment

- Hospital stay

- Incidence of retinopathy of prematurity

- Neonatal sepsis

\section{Box 5 Secondary biomarker outcomes}

Maternal plasma:

- Maternal plasma

- Soluble fms-like tyrosine kinase 1 (sFlt-1)

- Soluble endoglin

- Placental growth factor (PGF)

- Endothelin 1

- (These analytes will be measured in serial samples collected during expectant managements)

Placental samples:

- Haeme oxygenase-1

- sFlt-1

- Vascular endothelial growth factor

- PGF

- Hypoxia inducible factor-1 $\alpha$

- (mRNA and protein levels will be measured in these placental samples taken at delivery)

tested on a selected subgroup to determine if the pharmacokinetics are altered in pregnancy. Esomeprazole levels will also be determined in the cord blood to assess placental transfer. The placenta will be examined histologically, and we will quantify mRNA and protein expression of haeme oxygenase-1 (HO-1) and sFlt-1 and sEng will be measured (see box 5). HO-1 is an endogenous antioxidant protein that may be beneficial in pre-eclampsia. We postulate placental HO-1 will be upregulated by esomeprazole. mRNA will be quantified by quantitative PCR, and protein will be quantified by western blot and densitometric analysis of the band obtained on the gel blot.

\section{Data management and statistical analysis}

Data will be collected prospectively. Data entry and checking will be continuous and queries will be followed vigorously to ensure clarification without delay.

The analyses will be on an intention-to-treat principle with comparisons made between proton pump inhibitors and placebo, for primary and secondary outcomes. Comparisons will be expressed as relative risks or mean differences with $95 \%$ CIs.

The patient characteristics, by treatment group, will be presented as mean (SD), median (25th-75th centile), minimum, maximum and count (\%) depending on type and distribution. Significance level is set at 0.05 and all hypothesis testing will be two-sided.

The primary outcome will not be adjusted for multiple comparisons, but the secondary outcomes will be adjusted for multiple comparisons using a small number of prespecified outcomes. Two analyses are planned: (1) primary intention-to-treat analysis and (2) a treatment received analysis to examine response among those who actually took the tablets. The primary outcome (prolongation in days) between treatment groups will be tested using analysis of covariance regression analysis with both treatment group and gestational strata as 
covariates. If a fetus does demise during expectant management, we will give it a length of pregnancy prolongation of zero and will include it in the primary outcome analysis.

Results will be presented as mean group difference with $95 \%$ CIs. Standard regression diagnostics and transformation of primary outcome, to achieve adequate distributions of residuals, will be performed if indicated. Survival analyses (ie, time until delivery), using Cox proportional hazards regression and Kaplan-Meier survivorship curves will be used.

The secondary outcomes may be composite or single outcomes. Continuous variables will be compared using either $\mathrm{t}$ test (for normally distributed variables) or Mann-Whitney U (non-normally distributed). Categorical values will be compared using $\chi^{2}$ test. For the longitudinal data of plasma sFlt-1, sEng and endothelin 1 levels, we will (1) graph the data longitudinally, (2) compare levels between the groups relative to gestational age (3), compare levels between groups relative to days after recruitment and (4) compare levels between groups at delivery. Finally, the placental expression of sFlt-1, sEng and HO-1 will be compared using simple statistics.

\section{Adverse events}

Reporting and handling of adverse events and serious adverse events will be in accordance with the good clinical practice guidelines. ${ }^{17}$

\section{Unblinding}

Given the safety profile of esomeprazole in pregnancy has been well documented, we anticipate the need for unblinding will be very uncommon. However, we will have the following procedures in place should unblinding be required. Sheets with lists of 50 randomisation codes each will be sealed in individual, signed, numbered envelopes. If unblinding is requested, the relevant envelope will be opened by a person not involved in the trial in the presence of two witnesses, the group allocation read and the list resealed in an envelope and signed. The randomisation envelopes will be accessible at all times to the principal investigator who will be contactable by mobile telephone. The principal investigator will coordinate this process.

\section{Early termination of the trial}

If for any reason there is a need to stop the trial prematurely, this decision will be taken by the Data and Safety Monitoring Committee.

\section{DISCUSSION}

Pre-eclampsia is a serious life-threatening condition for both the mother and fetus and is associated with severe maternal and perinatal morbidity. If a treatment were to be discovered, it would have a major impact on both maternal and perinatal health. An ideal drug would ameliorate the biological disease process of preeclampsia in the mother and placenta, reduce the risk of serious complications from developing and allow pregnancies that were complicated by the disease at an early gestation to gain gestation without putting the mother and fetus at significantly increased risk.

However, there is currently no treatment besides delivery of fetus and placenta. Furthermore, there have been very few candidate-proposed treatments that have reached human trials. Thadhani $e t a l^{18}$ proposed the use of apheresis (or dialysis) to reduce the sFlt-1 fraction in the blood as a means to treat pre-eclampsia. That was a case series of eight patients where three had serial apheresis treatments with a possible prolongation of the pregnancy as a result. However, this is perhaps too invasive a treatment to be widely used to treat pre-eclampsia and its usefulness still remains unproven. As a result of preclinical studies, mainly on animal models, the anticholesterol drug pravastatin is being evaluated as a possible treatment for pre-eclampsia (STAMP trial, UK-based study). ${ }^{19}$ We are not aware of other significant trials of orally available small molecules to treat pre-eclampsia.

It is known that the pre-eclamptic placenta releases antiangiogenic sFlt-1 and sEng into the maternal circulation, causing widespread maternal endothelial dysfunction and organ injury. ${ }^{9}$ Pre-eclampsia is also associated with oxidative stress. A drug that can decrease sFlt-1 and sEng production, decrease endothelial dysfunction and oxidative stress may be a potential treatment for preeclampsia. We have generated preclinical data suggesting esomeprazole may have such actions. On the basis of this preclinical data, we are now proposing to undertake this phase II randomised clinical trial.

We propose recruiting 120 women at Tygerberg Hospital, in the Western Cape Provence of South Africa. There are two advantages to running the trial at this site. South Africa has a very high incidence of preeclampsia. Second, Tygerberg Hospital is a tertiary referral centre with a neonatal intensive care unit, a maternal critical care unit, an adult intensive care unit and an academic centre that actively contributes to the global scientific literature. Thus, we believe running this trial at Tygerberg Hospital represents a balance between obtaining sufficient number of cases of preterm pre-eclampsia, and offering modern obstetric and perinatal care, making the results potentially generalisable to both developed and developing countries.

Pravastatin is undergoing a randomised clinical trial in women with early onset pre-eclampsia (STAMP trial) and for women at risk of developing pre-eclampsia. ${ }^{19} 20$ However, pravastatin has been assigned to pregnancy category $\mathrm{X}$ by the Food and Drug Administration (FDA) categorisation system and database for prescribing medicines in pregnancy. The FDA classifies category X drugs in pregnancy as medications where studies in animals or humans have demonstrated fetal abnormalities and/or there is positive evidence of human fetal risk based on 
adverse reaction data from investigational or marketing experience, and the risks involved in use of the drug in pregnant women clearly outweigh potential benefits. ${ }^{21}$ Esomeprazole is classified as a category $\mathrm{C}$ drug in pregnancy by the FDA. Category $\mathrm{C}$ is for drugs where animal reproduction studies have shown an adverse effect on the fetus and there are no adequate and well-controlled studies in human, but potential benefits may warrant use of the drug in pregnant women despite potential risks. ${ }^{21}$ Esomeprazole may very well then be a safer option than pravastatin for treating pre-eclampsia.

Additional safety information on the use of proton pump inhibitors in pregnancy is also now available as they have been extensively used in pregnancy for the treatment of gastro-oesophageal conditions. Large population-based cohorts and systematic reviews (including administration in the first trimester) have not found any adverse effects in pregnancy and specifically, these studies have not shown any increased risk for congenital abnormalities, spontaneous miscarriage or preterm delivery. The most recent systematic literature review which was published in 2009 included 1530 pregnancies exposed to proton pump inhibitors and had 133410 non-exposed matched controls. ${ }^{10}$ The largest study to date, published in 2010, spanned a 13-year period and involved 840968 pregnancies of which 5082 were exposed to proton pump inhibitors in the first trimester of pregnancy. This study concluded that exposure to proton pump inhibitors, even during the first trimester of pregnancy, was not associated with an increased risk of major birth defects. ${ }^{12}$ In 2012, a further large study involving 112022 pregnancies of which 1186 pregnancies that had been exposed to proton pump inhibitors confirmed there was no associated increase risk for congenital anomalies with proton pump inhibitor use. Importantly this study also found no increase in fetal growth restriction or adverse neonatal outcomes (including premature delivery and low Apgar scores) in pregnancies exposed to proton pump inhibitors in the first, second or third trimesters of pregnancies. ${ }^{11}$ Thus, esomeprazole is likely to be safe in pregnancy. Furthermore, we will also only be using treatment for a relatively short period in the late second and early third trimesters, well past the time of organogenesis.

We have powered our study to the primary outcome, which is to show esomeprazole can safely prolong gestation for five days. We believe such a gain of gestation in preterm pre-eclampsia is likely to result in significantly better neonatal outcomes. However, a limitation in this trial is that we have not specifically powered to detect improvements in maternal, fetal or neonatal outcomes (although these are planned secondary outcomes). The trial would need to be significantly larger to detect such clinical improvements. Given esomeprazole has not been used to treat pre-eclampsia before, it seemed more pragmatic to first undertake the trial as planned. If this trial yields a positive result, a further phase III multicentre randomised trial may be required to be sufficiently powered to demonstrate improvements in clinical outcomes.
A further potential novelty of this trial is that we will measure biomarkers that reflect disease severity. We will measure circulating levels of sFlt-1 and sEng in serial samples obtained from the mother. These antiangiogenic factors are considered to play an important role in inciting maternal endothelial dysfunction and end-organ injury seen in pre-eclampsia. Their importance as biomarkers is highlighted by the fact that in the STAMP trial, the primary outcome is to examine whether pravastatin can decrease circulating sFlt-1 levels in the mother. Furthermore, we will measure expression of key molecules in the placental samples obtained at delivery.

Pre-eclampsia kills mothers, fetuses and neonates and is responsible for severe maternal and neonatal morbidity. This is especially the case in the developing world where there is a lack of resources, including staff, equipment and finances. Currently there is no treatment apart from delivery. If a treatment were to be discovered it would have dramatic effects on maternal and neonatal outcomes. Esomeprazole has shown potential as a therapeutic agent in preclinical work on pre-eclampsia. Further advantages of esomeprazole are that it is available in tablet form, is safe during pregnancy and is not expensive. This makes it an ideal candidate as a global therapeutic for preeclampsia. It is therefore imperative for this trial to be performed. If esomeprazole were proven to be effective at prolonging gestation in early onset pre-eclampsia it would be the first treatment option for this group of vulnerable mothers and could play an important role in decreasing the clinical burden of this dangerous condition.

\section{Author affiliations}

${ }^{1}$ Department of Obstetrics and Gynaecology, Stellenbosch University and Tygerberg Hospital, Cape Town, South Africa

${ }^{2}$ Translational Obstetrics Group, University of Melbourne, Mercy Hospital for Women, Melbourne, Victoria, Australia

${ }^{3}$ Department of Obstetrics and Gynaecology, Mercy Hospital for Women, University of Melbourne, Heidelberg, Victoria, Australia

${ }^{4}$ The Robinson Institute, School of Paediatrics and Reproductive Health, University of Adelaide, Adelaide, South Australia, Australia

${ }^{5}$ Department of Anaesthetics, Mercy Hospital for Women, University of Melbourne, Melbourne, Victoria, Australia

Acknowledgements The authors would like to acknowledge Professor Jane Norman and Justus Hofmeyr for their contributions to the protocol development.

Contributors CAC, SPW and ST developed and designed the trial. SPW and ST obtained funding for this trial. CAC, SPW and ST wrote the first draft of this manuscript. RH helped with the statistical aspects of this protocol. NH was involved in the design of the laboratory arm of this trial. All authors were involved in revision of the manuscript. All authors approved the final version to be submitted. CAC and ST responded to the reviewers comments.

Funding This work is supported by the Kilvington Trust, The Medical Foundation for Mothers and Babies and The Shiela Handbury Foundation. NHMRC provides salary support to ST and BWM.

\section{Competing interests None declared.}

Ethics approval This study has ethical approval (protocol number M14/09/ 038 Federal Wide assurance number 00001372, Institutional Review Board number IRB0005239) registered with NHREC (Application ID 3649) and the PACTR and has the South African Medical Control Council approval (MCC trial reference 20150309). 
Provenance and peer review Not commissioned; externally peer reviewed.

Data sharing statement The full protocol, patient information leaflet and consent forms are available as supplementary files. For further information please contact CAC by email: cathycluver@hotmail.com

Open Access This is an Open Access article distributed in accordance with the Creative Commons Attribution Non Commercial (CC BY-NC 4.0) license, which permits others to distribute, remix, adapt, build upon this work noncommercially, and license their derivative works on different terms, provided the original work is properly cited and the use is non-commercial. See: http:// creativecommons.org/licenses/by-nc/4.0/

\section{REFERENCES}

1. Lain KY, Roberts JM. Contemporary concepts of the pathogenesis and management of preeclampsia. JAMA 2002;287:3183-6.

2. ACOG Committee on Obstetric Practice. Practice bulletin\# 33: diagnosis and management of preeclampsia and eclampsia. Obstet Gynecol 2002;99:159-67.

3. Dolea C, AbouZahr C. Global burden of hypertensive disorders of pregnancy in the year 2000. Evidence and information for policy (EIP). Geneva: World Health Organization, 2003.

4. Redman CW, Sargent IL. Latest advances in understanding preeclampsia. Science 2005;308:1592-4.

5. Sibai B, Dekker G, Kupferminc M. Pre-eclampsia. Lancet 2005;365:785-99.

6. Sibai BM. Diagnosis and management of gestational hypertension and preeclampsia. Obstet Gynecol 2003;102:181-92.

7. Zhang J, Meikle S, Trumble A. Severe maternal morbidity associated with hypertensive disorders in pregnancy in the United States. Hypertens Pregnancy 2003;22:203-12.

8. United Nations Millenium Development Goals. http://www.un.org/ millenniumgoals/ (accessed Feb 2015).
9. Levine RJ, Maynard SE, Qian C, et al. Circulating angiogenic factors and the risk of preeclampsia. N Engl J Med 2004;350:672-83.

10. Gill SK, O'Brien L, Einarson TR, et al. The safety of proton pump inhibitors (PPIs) in pregnancy: a meta-analysis. Am J Gastroenterol 2009;104:1541-5.

11. Matok I, Levy A, Wiznitzer A, et al. The safety of fetal exposure to proton-pump inhibitors during pregnancy. Dig Dis Sci 2012;57:699-705.

12. Pasternak B, Hviid A. Use of proton-pump inhibitors in early pregnancy and the risk of birth defects. N Engl J Med 2010;363:2114-23.

13. Onda K, Hannan N, Beard S, et al. Proton Pump inhibitors for treatment of preeclampsia Abstract 6-OR. XIX Word Congress ISSHP; 26-29 Oct 2014.

14. Magee LA, Pels A, Helewa M, et al. Diagnosis, evaluation, and management of the hypertensive disorders of pregnancy. Pregnancy Hypertens 2014;4:105-45.

15. Hall DR, Odendaal HJ, Steyn DW, et al. Expectant management of early onset, severe pre-eclampsia: maternal outcome. BJOG 2000;107:1252-7.

16. Ryan TP. Sample Size Determination and Power. Hoboken, New Jersey: John Wiley and Sons, Inc; 2013.

17. World Health Organization. Guidelines for good clinical practice (GCP) for trials on pharmaceutical products. WHO Tech Rep Ser 1995;850:97-137.

18. Thadhani $\mathrm{R}$, Kisner $\mathrm{T}$, Hagmann $\mathrm{H}$, et al. Pilot study of extracorporeal removal of soluble fms-like tyrosine kinase 1 in preeclampsia. Circulation 2011:124:940-50.

19. Ramma W, Ahmed A. Therapeutic potential of statins and the induction of heme oxygenase-1 in preeclampsia. J Reprod Immunol 2014;101-102:153-60.

20. Costantine MM, Tamayo E, Lu F, et al. Using pravastatin to improve the vascular reactivity in a mouse model of soluble fms-like tyrosine kinase-1-induced preeclampsia. Obstet Gynecol 2010;116:114-20.

21. Teratology Society Public Affairs Committee. FDA classification of drugs for teratogenic risk. Teratology 1994;49:446-7. 